\title{
Modeling mitochondrial dysfunctions in the brain: from mice to men
}

\author{
Megan E. Breuer • Peter H. G. M. Willems • \\ Frans G. M. Russel • Werner J. H. Koopman • \\ Jan A. M. Smeitink
}

Received: 5 April 2011 /Revised: 20 June 2011 / Accepted: 27 June 2011 /Published online: 14 July 2011

(C) The Author(s) 2011. This article is published with open access at Springerlink.com

\begin{abstract}
The biologist Lewis Thomas once wrote: "my mitochondria comprise a very large proportion of me. I cannot do the calculation, but I suppose there is almost as much of them in sheer dry bulk as there is the rest of me". As humans, or indeed as any mammal, bird, or insect, we contain a specific molecular makeup that is driven by vast numbers of these miniscule powerhouses residing in most of our cells (mature red blood cells notwithstanding),
\end{abstract}

Communicated by: John Christodoulou

Competing interests: None declared.

M. E. Breuer • P. H. G. M. Willems • W. J. H. Koopman Department of Biochemistry, Nijmegen Centre for Molecular Life Sciences, Radboud University Nijmegen Medical Centre, Nijmegen, The Netherlands

\section{E. Breuer · J. A. M. Smeitink}

Department of Pediatrics, Nijmegen Centre for Mitochondrial

Disorders, Radboud University Nijmegen Medical Centre,

Nijmegen, The Netherlands

\section{F. G. M. Russel}

Department of Pharmacology and Toxicology,

Nijmegen Centre for Molecular Life Sciences,

Radboud University Nijmegen Medical Centre,

Nijmegen, The Netherlands

M. E. Breuer ' P. H. G. M. Willems • F. G. M. Russel •

W. J. H. Koopman · J. A. M. Smeitink

Centre for Systems Biology and Biochemistry,

Nijmegen Centre for Molecular Life Sciences,

Radboud University Nijmegen Medical Centre,

Nijmegen, The Netherlands

\section{E. Breuer $(\square)$}

Department of Biochemistry, Nijmegen Centre for Mitochondrial

Diseases, Radboud University Nijmegen Medical Centre,

P.O. Box 9101, NL-6500 HB Nijmegen, The Netherlands

e-mail: M.Breuer@cukz.umcn.nl quietly replicating, living independent lives and containing their own DNA. Everything we do, from running a marathon to breathing, is driven by these small batteries, and yet there is evidence that these molecular energy sources were originally bacteria, possibly parasitic, incorporated into our cells through symbiosis. Dysfunctions in these organelles can lead to debilitating, and sometimes fatal, diseases of almost all the bodies' major organs. Mitochondrial dysfunction has been implicated in a wide variety of human disorders either as a primary cause or as a secondary consequence. To better understand the role of mitochondrial dysfunction in human disease, a multitude of pharmacologically induced and genetically manipulated animal models have been developed showing to a greater or lesser extent the clinical symptoms observed in patients with known and unknown causes of the disease. This review will focus on diseases of the brain and spinal cord in which mitochondrial dysfunction has been proven or is suspected and on animal models that are currently used to study the etiology, pathogenesis and treatment of these diseases.

$\begin{array}{ll}\text { Abbreviations } \\ \text { 2DG } & \text { 2 deoxyglucose } \\ \text { 3-NP } & \text { 3-nitroproprionic acid } \\ \text { 6-OHDA } & \text { 6-hydroxydopamine } \\ \text { AD } & \text { Alzheimer's disease } \\ \text { AiF } & \text { Apoptosis inducing factor } \\ \text { ALCAR } & \text { Acetyl L-carnitine } \\ \text { ALS } & \text { Amyotrophic lateral sclerosis } \\ \text { ATP } & \text { Adenosine triphosphate } \\ \text { BDNF } & \text { Brain-derived neurotrophic factor } \\ \text { CO } & \text { Cytochrome oxidase } \\ \text { DA } & \text { Dopamine } \\ \text { DOPAC } & \text { 3,4-dihydroxy-phenylacetic acid } \\ \text { GABA } & \text { Gamma aminobuteric acid }\end{array}$




$\begin{array}{ll}\text { HD } & \text { Huntington's disease } \\ \text { HPA axis } & \text { Hypothalamus-pituitary-adrenal axis } \\ \text { HVA } & \text { Homovanillic acid } \\ \text { MAO-B } & \text { Monoamine oxidase-B } \\ \text { MPTP } & \text { 1-methyl 4-phenyl 1,2,3, } \\ & \text { 6-tetrahydropyridine } \\ \text { MPP } & \text { 1-methyl 4-phynylpyridine } \\ \text { MSSN } & \text { Medium sized spiny neurons } \\ \text { mtDNA } & \text { Mitochondrial DNA } \\ \text { MOMP } & \text { mitochondrial outer membrane } \\ & \text { permeabilization } \\ \text { NAD } & \text { Nicotinamide adenine dinucleotide } \\ \text { NMDA } & \text { N-methyl-D-aspartic acid } \\ \text { PD } & \text { Parkinson's disease } \\ \text { PEO } & \text { Progressive external ophthalmoplegia } \\ \text { PUFA } & \text { Polyunsaturated fatty acid } \\ \text { ROS } & \text { Reactive oxygen species } \\ \text { SNpc } & \text { Substantia nigra pars compactica } \\ \text { SOD1 } & \text { Superoxide dismutase 1 } \\ \text { TCA } & \text { Tri-cyclic antidepressant } \\ \text { UPS } & \text { Ubiquitin-proteasome } \\ \text { VDAC } & \text { Voltage dependent anion channels } \\ \text { YAC } & \text { Yeast artificial chromosome }\end{array}$

\section{Introduction}

It has long been known that the energy source for all eukaryotic cells is the organelle called the mitochondrion. These organelles, thought to be incorporated as bacteria into our cells at some point in our evolution, contain their own DNA, inherited from our mothers, and replicate through cloning, fission and fusion. There has been some debate as to whether or not the original symbiosis between mitochondrion and cell was more beneficial to the mitochondria, or more damaging to the cell in which it resides (Searcy 2003), but it is generally agreed that cells receiving their energy from mitochondria are infinitely more successful than bacterial cells, which generate their energy without them. Mitochondria serve multiple purposes in our bodies, among which include the modulation of both cell proliferation and programmed cell death (Carreras et al. 2007). One of the most important functions of the mitochondria is to convert energy from macronutrients (fatty acids, carbohydrates and amino acids) to ATP, through the oxidation of the macronutrients, reducing oxygen to water, and phosphorylating adenosine diphosphate (ADP) to ATP (Schiff et al. 2011)(For a more in depth description of oxidative phosphorylation, please see Boveris and Navarro 2008). The mitochondrial respiratory chain is found in the mitochondrial inner membrane, and consists of four complexes. Reduced equivalents from sugar-derived organic acids, from amino acids, or from fatty acids enter the chain via dehydrogenases (complexes I and II), and are then subsequently transferred to complexes III and IV. With each transfer, electrons release energy into the intermembrane space, creating a proton gradient. The flow of protons back through complex $\mathrm{V}$ is responsible for the generation of ATP; complex V is also responsible for the release of energy stored in the proton gradient by facilitating proton flow into the mitochondrial matrix (Clay et al. 2010). However, defects in mitochondrial function, especially in respiration, can lead to debilitating, possibly fatal, abnormalities. For example, mitochondrial dysfunction has been linked to such neurodegenerative and neurological diseases as Parkinson's, Alzheimer's and Huntington's, as well as amyotrophic lateral sclerosis (ALS), schizophrenia and depression, and can also result in growth retardation, optic atrophy, hypertrophic cardiomyopathy, and metabolic disorders (Levine et al. 2004; Ben-Shachar 2009; Clay et al. 2010). This review will examine the health implications of mitochondrial dysfunctions, specifically focusing on brain aberrations, how these abnormalities have been modeled in vivo and how symptoms may be improved through either pharmacological or nutritional interventions. Since there is an excess of information about numerous knockout, knock-in, transgenic and induction animal models, only those that have been examined in behavioral paradigms, with or without pharmaceutical or nutraceutical interventions, will be discussed here.

\section{Mitochondrial dysfunction: the human brain and spinal cord}

Disruptions in mitochondrial function can have severe consequences, not the least of which is neurodegeneration in the brain. The production of damaging reactive oxygen species (ROS) by dysfunctional mitochondria may lead to increases in oxidative stress and subsequent neuronal death. The brain is extremely susceptible to oxidative stress, because it consumes significant amounts of oxygen, but has fewer defenses against free radicals (Weinstock and Shoham 2003). Further explanations as to the brain's sensitivity to ROS include the fact that the brain is rich in polyunsaturated fatty acids (PUFAs), which are highly susceptible to ROS damage (Cui et al. 2004). Further, the release of excitatory neurotransmitters, such as glutamate, begins a chain of reactions in the postsynaptic neurons, which also result in the formation of ROS (Cui et al. 2004). However, the underlying mitochondrial cause for neurodegenerative diseases does not only include the production of ROS. Other factors, such as altered calcium flux and energy generation dysfunctions can make significant contributions. For example, events such as mitochondrial outer membrane permeabilization (MOMP) 
can lead to mitochondrial respiratory dysfunctions, resulting in the release of such proteins as Apoptosis Inducing Factor (AiF) and cytochrome c. Since neurons in the brain are highly sensitive to changes in mitochondrial respiration, changes in MOMP may ultimately lead to neurodegeneration (Green and Kroemer 2004). Furthermore, mitochondria have a large capacity for calcium uptake and are integral for calcium storage in cells. Calcium flux is regulated by the mitochondrial inner membrane potential; disturbances in calcium flux may result in increased calcium uptake by the mitochondria, resulting in apoptosis and neuronal death (Andrews et al. 2005).

There is increasing evidence that these disruptions in mitochondrial function and increased oxidative stress may play a role in the development of neurodegenerative diseases such as Parkinson's (PD), Alzheimer's (AD), Huntington's (HD), and ALS (Lin and Beal 2006), as well as the development of depression, anxiety, and schizophrenia (Scaglia 2010)(Table 1). Aging also plays a significant role in the development of neurodegenerative diseases, as mitochondria are thought to speed the process of senescence through the accumulation of mitochondrial DNA (mtDNA) mutations, which correlate with declining mitochondrial function and subsequent neurodegeneration (Lin and Beal 2006). For a more in depth overview of mitochondrial dysfunction, oxidative stress and the roles they play in neurodegenerative diseases, please see Lin and Beal 2006.

Whatever the cause, the fact remains that disruptions in mitochondrial functioning have significant effects on the development of neurodegenerative diseases. The first evidence for this was found in the unlikely source of drug abusers: those who were accidentally exposed to the drug 1-methyl 4-phenyl 1,2,3,6-tetrahydropyridine (MPTP), the metabolite of which (MPP) is extremely neurotoxic (and also happens to be a complex I inhibitor), developed PD (Langston et al. 1983; Choi et al. 2008; Schapira 2010).

\section{Parkinson's disease}

Most often, neurons in the motor centers of the brain, such as the cerebellum and striatum, have been found to be decreased in PD patients, along with a dramatic decrease of dopamine neurons in the substantia nigra pars compactica $(\mathrm{SNpc})$ and the generation of proteinaceous inclusions known as Lewy bodies, also in dopamine (DA) neurons (Tansey and Goldberg 2010). However, patients presenting with PD often show multiple symptoms and brain histology points to several causes, not just the depletion of dopaminergic neurons. Decreases in Purkinje cell numbers in the cerebellum have also been observed in the brains of patients with neurodegenerative diseases, correlating with a loss of motor skills (Mounsey and Teismann 2010).
Symptoms of PD include tremor, rigidity, bradykinesia (slowed movement), and postural instability (Xiong et al. 2009). The cause of PD is at this point still relatively unknown as most cases have no genetic links, but it is thought that dysfunctions in mitochondrial respiration, especially in complex I, result in increased ROS production, oxidative stress and dopaminergic neuronal degeneration, all of which are associated with the development of PD (de Moura et al. 2010).

\section{Huntington's disease}

The neurodegenerative basis for HD is characterized by multiple "CAG" replications in the huntingtin gene. Those affected begin to experience decreased motor function and eventually descend into the gloom of severe cognitive impairment (Novak and Tibrizi 2010). The more CAG copies present, the faster the disease progression, and there is no cure. The hallmarks of HD include widespread nuclear inclusions in the brain and significant decreases in medium sized spiny neurons (MSSN) in the striatum (Levine et al. 2004). However, even though there is evidence that mitochondrial dysfunction may play a significant role in the development of HD, the precise role played by the mitochondria in the disease pathology is unknown (Oliveira 2010). Post mortem examination of brains of advanced HD sufferers does show reduced in vitro activity of mitochondrial complexes II, III and IV (Browne et al. 1997). Aconitase activity has also been shown to be significantly decreased in HD sufferers (as well as in the striatum of R6/2 knock in mice, an in vivo model for HD), further underlining the importance of mitochondrial dysfunction in the development of this disease, since aconitase is particularly vulnerable to free radicals (Chen 2011). However, those with pre-symptomatic or early cases of HD did not show mitochondrial respiration deficiencies, and striatal oxygen metabolism appeared to be normal (Oliveira 2010).

\section{Alzheimer's disease}

The hallmarks of Alzheimer's disease include severe decreases in memory, accompanied by deterioration of intellectual function, personality changes, and inability for self care (Masliah et al. 1994). In the brain, the diseases manifests in the form of neurofibrillary tangles comprised mainly of hyperphosphorylated tau proteins and extracellular senile plaques composed of $\beta$-amyloid and mostly found on cholinergic neurons supplying the hippocampus (Dragicevic et al. 2010). Disruptions in mitochondrial respiration have been thought to play a role in the development of the disease, as rats injected with the drug sodium azide, a selective complex IV inhibitor, show cognitive deficits 


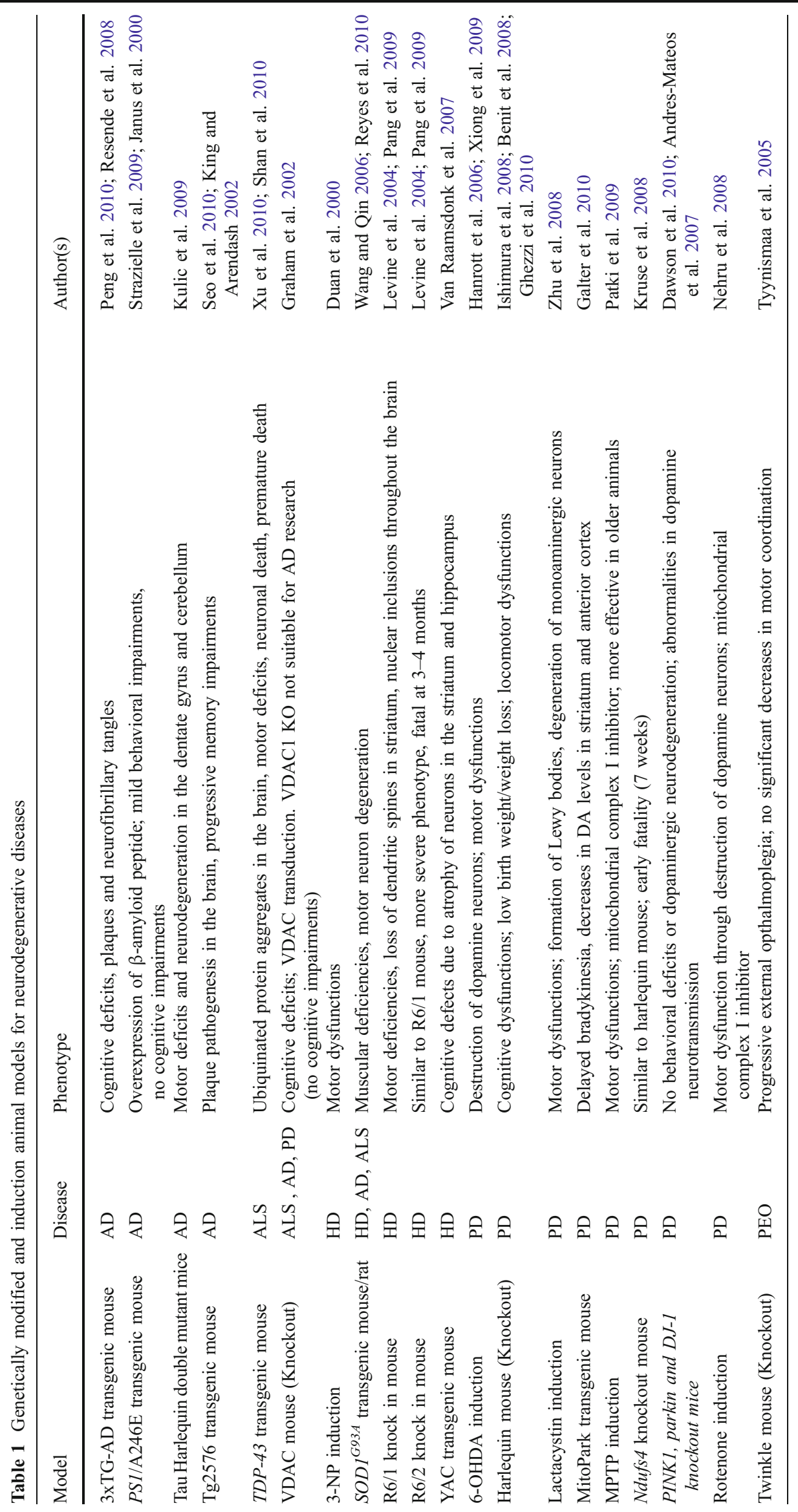


similar to those suffering AD (Bennett et al. 1996). Complex IV plays an important role in energy metabolism and the production of adenosine tri-phosphate (ATP) (Hirai et al. 2001; Smeitink et al. 2001).

\section{Amyotrophic lateral sclerosis}

Amyotrophic lateral sclerosis (ALS), also known as motor neuron disease and Lou Gehrig's disease, has no known cause outside of the few familial cases resulting from mutations in the superoxide dismutase 1 (SOD1) gene. SOD1, a soluble cytoplasmic and mitochondrial intermembrane space protein, is responsible for the conversion of superoxide into oxygen and hydrogen peroxide, protecting the cell from superoxide toxicity. ALS is characterized by cognitive and motor deficits, with a loss of motor neurons in the cortex, brain stem and spinal cord, as well as neurofilament aggregates in the proximal axons of motor neurons. The hallmarks of ALS, known as Bunina bodies, are eosinophilic bodies found in the soma of anterior horn cells (Goodall and Morrison 2006). More recently, it has been discovered that mutations in TARDBP, the gene encoding the transactivation response DNA-binding protein TDP-43, results in an ALS phenotype in transgenic mice (TDP- $\left.43_{\text {PrP }}\right)$. This protein is the main component of ubiquinated aggregates in the brain and causes the loss of motor neurons, resulting in ALS symptoms and ultimately, premature death (Xu et al. 2010; Shan et al. 2010). Shan et al. (2010) also point out that mutations in the FUS/TLS gene, encoding for another RNA-binding protein, is linked to ALS, suggesting that alterations in RNA metabolism or processing may also contribute to neurodegeneration. There is no effective treatment for ALS to date, and most of those diagnosed will succumb to the disease within 3 to 5 years after diagnosis (Fornai et al. 2008).

Neuropsychiatric diseases

Mitochondrial dysfunction has also been implicated in the development of several neuropsychiatric diseases such as depression, anxiety and schizophrenia. There is evidence that dysfunctions in mitochondrial respiration have severe consequences for monoamine levels in the brain, resulting in decreases in serotonin (5-hydroxytryptamine, or 5-HT), dopamine, noradrenaline, and gamma aminobuteric acid (GABA)(Gardner and Boles 2010), all of which are monoamines associated with depressive, schizophrenic and anxious behaviors. For example, ties have been found between mitochondrial dysfunction and the development of schizophrenia: microscopic analysis of autopsy specimens show mitochondrial malformations and reduced density in the anterior limbic cortex and the caudate putamen nucleus, both areas highly associated with the development of schizophrenia (Ben-Shachar 2009). Mitochondrial dysfunction has also been linked to decreases in brain-derived neurotrophic factor (BDNF), decreases in hippocampal neurons and disruption of the hypothalamus-pituitaryadrenal (HPA) axis, all of which has been linked to the development of depressive and anxious symptoms (Clay et al. 2010; Wu et al. 2010).

The scope and severity of the above named disorders makes it clear that much work needs to be done examining the causes and treatments for neurodegenerative and psychiatric disorders, perhaps with a stronger focus on mitochondrial dysfunction. One of the most often used and valid ways to accomplish this goal is through the use of animal models. Currently, several animal models exist that specifically replicate certain aspects of many of the aforementioned diseases. The use of these models is key to the development of new and improved treatments, and may even lead to more clues as to the connections between mitochondrial dysfunction and neurodegenerative diseases.

\section{In vivo modeling of mitochondrial dysfunction}

While several breakthroughs have been made as to understanding the reactions of isolated mitochondria to various stimuli under in vitro conditions, it is important to understand the working mechanisms of these organelles in vivo. Animal models are extremely useful, and even necessary, when observing the effects of mitochondrial respiration and its effects on brain function (Table 2). Indeed, without the use of these animal models, finding treatments for mitochondrial diseases would be next to impossible.

Mouse models for mitochondrial complex I deficiencies

\section{Ndufs4 knockout mouse}

With the advent of genetic engineering, it has become possible to generate specific animal models for diseases. Along these lines, the $N d u f_{s} 4$ mouse knockout was generated for the purpose of examining mitochondrial complex I disorders. In short, inactivation of the $N d u f_{s} 4$ gene directly effects the encoding of mitochondrial complex I (Kruse et al. 2008), resulting in a phenotype that includes many of the symptoms seen in humans suffering the same affliction: low birth weight, followed by subsequent failure to thrive, partial to total blindness, and impairments in motor function; these mice often die about seven weeks after birth. Knockout mice also exhibit abnormal mitochondrial morphology in the soleus muscle, characterized by swollen mitochondria containing condensed cristae (Quintana et al. 2010; Kruse et al. 2008). Puzzlingly, these mice exhibit 
normal muscular oxygen consumption and ATP levels and have normal fasting blood glucose levels (Kruse et al. 2008). Therefore, it must be assumed that the cause of early death in these animals is not directly dependent on mitochondrial respiration abnormalities, but perhaps is more connected to the sensitivity of the brain to oxidative stress. Since the brain is less adept at protecting itself from free radicals, it may be that these animals are more susceptible to neurological ROS damage. Indeed, these animals show neurodegeneration from an early age (approximately 5 weeks), as seen from their decreased performance in the rotarod and rope grip paradigms, as well as cognitive dysfunctions. Knockout animals show decreases in Purkinje cells in the cerebellum, and neuronal damage in the striatum and hippocampus (Kruse et al. 2008). The fact that these animals have significantly decreased body temperatures (up to $4^{\circ} \mathrm{C}$ less than wild types) and have significantly increased responses in a startle paradigm may also indicate compromises in their brain monoamine system, particularly serotonin. It may be that mitochondrial dysfunctions in these animals also leads to disruptions in their homeostasis, resulting in decreased body temperature, altered appetite, and disrupted circadian rhythms.

Interestingly, others examining in vivo $N d u f s 4$ mutations have found an embryonically lethal phenotype in knockout animals, while heterozygotes show more of the behavioral characteristics typically found in the knockouts in other labs (Ingraham et al. 2009). However, this group, instead of knocking out the entire NDUFS4 protein, only eliminated the last 10-15 amino acids, which may account for the lethal phenotype of their knockout animals.

\section{The harlequin mouse}

This particular knockout mouse was also generated to study the effects of mitochondrial dysfunction on disease progression (Ishimura et al. 2008). The mutation is a result of a proviral insertion in the X-linked gene encoding the mitochondrial protein Apoptosis Inducing Factor (AiF) (Benit et al. 2008). This causes a $20 \%$ decrease of $\mathrm{AiF}$ compared to wild types, and results in characteristics that are similar to the $N d u f_{S} 4$ mouse, but show a generally more stable phenotype, allowing it to live significantly longer than $N d u f s 4$ s. These animals also show fur loss around about three months of age, severe cerebellar ataxia, optic tract dysfunctions, and have an increased risk of hypertrophic cardiomyopathy (Benit et al. 2008). Similarly to the Ndufs 4 knockout, these animals show severe deficits on the rotarod test for motor coordination. Since the mutation is X-linked, the majority of animals showing abnormalities are male, and then only about $50 \%$ show growth abnormalities. However, almost all animals, males and females, are ataxic at about 3 months; $90 \%$ of the 
heterozygotes have gait ataxia at this age (Benit et al. 2008). Brain pathology at six months of age reveals that complex I activity is significantly decreased in the cerebellum, thalamus and cortical enriched fraction, as well as in the optic nerves and retinas, compared to wild types. However, complex I activity is normal in the heart, liver and testes, and decreased by $30 \%$ in skeletal muscle (Benit et al. 2008). It is interesting that two different animal models, both examining the effects of complex I function, should differ from each other so drastically in life expectancy and pathology. The AiF mutation has been hypothesized to result in an independent pathogenic mechanism, responsible for destabilization of the inner mitochondrial membrane. This destabilization leaves the respiratory chain and its functions open to damage, leading to the deficiencies in complex I function (Ghezzi et al. 2010). However, the exact mechanism behind the effects of AiF on the mitochondrial respiratory chain in both humans and the Harlequin mouse remains unknown.

\section{Models for Parkinson's disease}

\section{The Mitopark mouse}

The MitoPark mouse is a transgenic model for Parkinson's disease, in which the mitochondrial transcription factor Tfam is selectively removed from midbrain dopamine neurons. Neurons lacking Tfam develop severe mitochondrial respiratory chain deficiencies and ultimately undergo cell death (Galter et al. 2010) resulting in a slow progression of PD symptoms as seen in human patients. MitoPark mice are generated through crossing knock in mice expressing Cre recombinase (driven by the dopamine transporter promoter) with mice in which the Tfam gene is flanked by loxP recombination sites, creating a model in which Tfam is only absent from neurons that express the dopamine transporter (Galter et al. 2010). When tested in an open field environment, these animals show decreases in horizontal (walking) and vertical (rearing) behaviors, starting at approximately 24 weeks of age. Interestingly, these animals also show decreases in dopamine, DOPAC and HVA levels in the striatum and anterior cortex (as measured by microdialysis), corresponding with the behavioral data, and also mimicking human patients (Galter et al. 2010). The bradykinesia seen in these animals also responds well to treatment with the dopamine precursor levodopa (L-DOPA) at a doses of 4 and $20 \mathrm{mg} / \mathrm{kg}$ (Galter et al. 2010), further underlying the validity of this particular animal model for PD.

parkin, PINK1 and DJ-1 mice

parkin, PINK1 and DJ-1 are three knockout mouse models for autosomal-recessive PD (for review, see Dawson et al.
2010). More than 100 mutations in parkin have been reported in PD patients and account for $50 \%$ of familial cases of PD; PINK1 mutations are recessively inherited. Both parkin and PINK1 knockout mice do not exhibit any abnormalities in dopamine levels or dopaminergic neurons, nor do they show dysfunctions in locomotion. However, both parkin and PINK1 mice show deficits in nigrostriatal dopamine transmission and have mild mitochondrial defects (Dawson et al. 2010).

DJ-1 is a member of the ThiJ/Pfpl family of molecular chaperones; it is protective against oxidative stress in mitochondria and plays a small role in early-onset PD (Gasser 2009). DJ-1 knockout mice do not exhibit any major behavioral abnormalities, and dopamine neuron numbers and striatal dopamine levels remain normal. However, neurotransmission in the nigrostriatal circuit and mitochondrial dysfunction has been observed in some $D J-1$ animals, similar to PINK1 and parkin knockouts (AndresMateos et al. 2007). It seems therefore that these three genetic animal models for PD may only represent symptoms associated with the onset of the disease, and are not suitable for studies examining the efficacy of treatments after substantial neurodegeneration has occurred.

\section{Rotenone, MPTP, 6-OHDA and lactacystin} induction models

Rotenone is a powerful mitochondrial complex I inhibitor, and has been used as an induction model for many neurodegenerative diseases (Chou et al. 2010). Rotenone easily passes through the blood brain barrier and cell membrane, due to its lipophilic structure (Nehru et al. 2008). Once absorbed, it can cause the destruction of dopamine neurons and results in PD-like symptoms and behaviors (Alam et al. 2004). In the brain, this induction model results in increased iron concentrations, which may lead to hydroxyl radical formation. Rotenone also causes decreases in the thiol antioxidant glutathione (GSH), which is responsible for peroxide clearance from the brain, and causes oxidative damage to lipids, proteins, and DNA in the substantia nigra (Vali et al. 2007). This pathology is similar to the post-mortem brains of PD sufferers, and therefore is a popular model for PD research in vivo.

The drug 1-methyl 4-phenyl 1,2,3,6-tetrahydropyridine (MPTP) is also a complex I inhibitor, and works by inhibiting oxidative phosphorylation of complex I, increasing ROS production, and decreasing ATP (Patki et al. 2009). MPTP may trigger neurodegeneration through the destruction of dopamine neurons in the SNpc, considered as one of the defining characteristics of PD.

Administration of 6-hydroxydopamine (6-OHDA) also results in the widespread deterioration of dopaminergic 
neurons in the nigrostriatal pathway, but does not result in the generation of Lewy bodies or altered $\alpha$-synuclein expression, as seen in PD patients (Xiong et al. 2009). The working mechanism of this neurotoxin is not exactly known, but it is thought that administration of 6-OHDA results in extracellular auto-oxidation and the induction of oxidative stress from the resulting oxidative products (Hanrott et al. 2006).

Exposure to the ubiquitin-proteasome (UPS) inhibitor lactacystin results in a phenotype similar to that of PD patients by causing noradrenaline, serotonin and dopamine neurons to degenerate and Lewy body accumulations when injected into the median forebrain bundle (Zhu et al. 2008). However, none of these induction models result in the complete spectrum of the characteristic chronic disease symptomologies. Therefore, while induction models are highly useful when examining neurological diseases, better models are needed which encompass the entire scope of the disease progression.

\section{Models for Huntington's disease}

\section{R6/1 and R6/2 knock-in and YAC transgenic mice}

There are many particular types of mouse models for Huntington's disease, including mice that contain only a fragment of exon-1 of the human huntingtin gene containing polyglutamine mutations (this in addition to both alleles of murine wild-type huntingtin, $\mathrm{Hdh}$ ), mice with pathogenic CAG repeats inserted into existing CAG expansion in the murine huntingtin Hdh (knock ins), and mice that express the full-length human HD gene (including murine Hdh) (Adhihetty and Beal 2008; Wang and Qin 2006). One such strain, called R6/2, generated with approximately $155 \mathrm{CAG}$ repeats inserted into their huntingtin genes, show subtle motor deficiencies and learning deficits one month after birth, with symptoms becoming more pronounced at two months, and becoming fatal at three to four months (Levine et al. 2004). The brain pathology of these mice shows nuclear inclusions present throughout the brain, and can be detected as early as three weeks after birth. Importantly, these inclusions have also been observed in the post mortem brains of HD patients. Also, R6/2 mice show loss of dendritic spines, as well as decreases in spine and dendrite numbers, in the striatal and cortical somatodendritic areas (Levine et al. 2004). This correlates with the theory that early degeneration of the cortical-striatal pathway can occur with the accumulation of huntingtin protein in cortical neurons (Tang et al. 2007). MSSNs in particular are more sensitive to N-methyl-D-aspartic acid (NMDA), an amino acid derivative that mimics glutamate's working mechanism (and therefore has effects on MSSNs susceptibility to excitotoxicity); NMDA-induced cellular swelling is indeed increased in the R6/2 knock-in (Levine et al. 1999). The R6/2 mouse has therefore become a popular model for testing putative new treatments for HD. These therapies include those that work on excitotoxicity (remacemide, riluzole), mitochondrial functioning (coenzyme Q, creatine), aggregate formation (Congo red, trehalose) and transglutaminases and caspases such as cystamine and minocycline, respectively (Levine et al. 2004). Several of these treatments prolonged survival and/or delayed the appearance of motor deficiencies in the R6/2 mouse.

R6/1 mice, similar to R6/2, show the same symptomology, but with a slower disease progression and milder phenotype. Interestingly, studies with this knock-in show that not only do these mice exhibit the traditional symptomology of HD, but also co-morbid depressive symptoms, as seen in the tail suspension and Porsolt forced swim paradigms (Pang et al. 2009). These depressive behaviors were ameliorated through cage enrichment, wheel running, and treatment with the selective serotonin reuptake inhibitor (SSRI), sertraline, but this study also showed that there were marked differences between male and female animals, stressing the importance of gender selection when performing behavioral studies (Pang et al. 2009).

Yeast artificial chromosome (YAC) mice were created to specifically model the CAG repeating aspect of HD, and may have 46,72 or 128 CAG repeats. The corresponding Huntington's symptomology is similar to patients with $\mathrm{HD}$, in that animals with more repeats show a more severe phenotype (Van Raamsdonk et al. 2007). YAC128 mice show atrophy in several brain regions, including the striatum, hippocampus and cerebellum. These animals also develop severe cognitive deficits, altered pre-pulse inhibition, and are at first hyperactive in an open field environment, but later become hypoactive. YAC 72 and 46 mice show similar deficits, but to a lesser extent, and later in life, similar to human HD patients (Van Raamsdonk et al. 2007).

\section{3-NP induction model}

The complex II and succinate dehydrogenase inhibitor 3nitropropionic acid (3-NP) is used as an induction model for HD; after exposure, animals show motor dysfunctions through degeneration of striatal neurons, similar to patients suffering from HD (Duan et al. 2000). This compound is thought to generate (indirect) excitotoxicity through the stimulation of NMDA receptors in the striatum, but this remains a matter of some controversy (Kim et al. 2000). 
Models for amyotrophic lateral sclerosis (ALS)

SOD1 ${ }^{G 93 A}$ transgenic mice and rats

These two genetically manipulated rodent strains are not only used for the study of ALS, but are also useful in vivo models for HD and PD. Transgenic animals expressing SOD $1^{\mathrm{G} 93 \mathrm{~A}}$ carry a mutated, mis-folded form of the human gene for SOD1. Energy deprivation following the impairment of the mitochondrial respiratory chain, alterations in intracellular calcium handling and activation of the apoptotic pathway results in the subsequent destruction of mitochondria in the spinal cord and the degeneration of motor neurons and astrocytes in cortical and spinal cord/brainstem areas (Israelson et al. 2010; Reyes et al. 2010; Lenzken et al. 2011). This mutation leads to gait abnormalities, as seen on the rotarod paradigm, deficiencies in grip strength and a truncated life span (Gurney et al. 1994; Lenzken et al. 2011).

\section{TDP-43 transgenic mice}

$T D P-43$, a DNA/RNA binding protein, played an unknown role in the development of neurodegenerative diseases, such as ALS, until recently. The role played by TDP-43 in ALS pathology became largely significant when it was shown that mutations in this gene resulted in ubiquinated protein aggregates as seen in many cases of ALS. TDP-43 mice show developmental retardation, motor deficits and premature death (Shan et al. 2010; Xu et al. 2010). Interestingly, mice that were bred with higher numbers of transgene copies showed a more severe phenotype. For example, animals from the W1 line, with the highest number of copies, were significantly smaller compared to nontransgenic littermates, and died at three weeks of age. Those animals from lines W2 and W3, with lower numbers of transgene copies, showed a less severe phenotype and survived to adulthood. However, male animals from these two lines showed a significantly more severe phenotype compared to female transgene littermates. Female TDP-43 mice showed no significant reductions in body weight and developed tremors only at 3 months of age, further underlying the importance of gender selection when performing in vivo behavioral studies (Shan et al. 2010).

Models for Alzheimer's disease

\section{PS1/A246E, Tg2575 and 3xTG-AD transgenic mice}

The PS1/A246E transgenic mouse is used as a model for autosomal dominant, early onset Alzheimer's disease, and is often used to examine early neurochemical changes associated with the disease (Strazielle et al. 2009). Mutations in the presenilin 1 (PS1) gene lead to increased production of the amyloidogenic form of $A \beta$ peptide. The neurotoxic properties of this increased $A \beta$ peptide may lead to increased oxidative stress and therefore increased mitochondrial damage (Wang et al. 2005). This particular mouse model does not, however, show severe cognitive impairments, and shows comparable cognition performance compared to controls in the Morris water maze (Janus et al. 2000).

The popular $\operatorname{Tg} 2576$ murine model of Alzheimer's disease, generated by over-expressing a mutated form of human A $\beta P P(\mathrm{~K} 670 \mathrm{M} / \mathrm{N} 671 \mathrm{~L})$, is often used in studying $\mathrm{AD}$ because it exhibits plaque formation in the brain and progressive memory impairments, similar to those suffering AD (Seo et al. 2010). More specifically, cognition testing in these animals reveals that they show deficits in the Morris water maze, the Y-maze and several kinds of passive avoidance paradigms (King and Arendash 2002). Also, unlike many of the animal models named previously, this particular model is widely commercially available and therefore easily obtainable for those wanting an in vivo $\mathrm{AD}$ model. The AD-like pathology shown by these animals is thought to be due to the transgenic expression of a mutant form of human A $\beta$ PP in the brain, which may also have effects on neurons in the spinal cord, which may also partially account for the motor dysfunctions seen in these animals (Seo et al. 2010).

The triple mutant mouse 3xTG-AD expresses the mutant genes $\mathrm{APP}_{\text {swe }}, \mathrm{PS}_{\mathrm{M}_{146 \mathrm{~V}}}$ and tau $301 \mathrm{~L}$, and exhibits not only the cognitive deficits seen with $\mathrm{AD}$, but also show the characteristic extracellular senile plaques and neurofibrillary tangles associated with the disease (Peng et al. 2010). These mice also show decreases in vitamin $\mathrm{E}$ and glutathione (two non-enzymatic antioxidants) levels, suggesting that these animals are much more susceptible to oxidative stress due to their decreased levels of endogenous antioxidants (Resende et al. 2008).

\section{Tau/Harlequin double mutant mice}

These mice were generated by crossing the Harlequin mouse, whose genetic makeup has been previously discussed, with mice expressing human mutant tau tg (P301L), a mouse model for human tau pathology seen in AD. These mice express the longest isoforms of human tau, together with the pathogenic mutation P301L, a mutation in the human Mapt gene encoding tau protein (Attems et al. 2011). The combination of these two strains results in a mouse model that shows increases in tau pathology, apoptotic neurodegeneration, and muscular/ motor deficits (Kulic et al. 2009). This model may be 
particularly interesting in the study of the connections between mitochondrial dysfunction and $\mathrm{AD}$, as it incorporates the genetic makeup of the harlequin mouse (including the AiF mutation), combining the neurodegenerative aspects of complex I dysfunction with the tau pathology found in $\mathrm{AD}$ patients.

\section{VDAC deficient mouse}

Voltage-dependent anion channels (VDAC) are proteins that form a voltage-gated ion channel in the mitochondrial outer membrane, allowing translocation of metabolites across the membrane (Graham et al. 2002). Humans and mice have three isoforms (VDAC1, VDAC2 and VDAC3). Mouse mutants generated for the VDAC1, VDAC 3 and VDAC 1/3 isoforms showed significant deficits in learning and memory paradigms (Weeber et al. 2002; Graham et al. 2002). When knockout mice for all three of these isoforms were tested in the Morris water maze, all three exhibited deficits in spatial learning, and did not exhibit any motor control or nociceptive deficits (Weeber et al. 2002). Electrophysiological examination of these animals revealed decreased plasticity of hippocampal neurons, further underlining the effects of VDAC deficiency and subsequent mitochondrial respiratory chain dysfunctions on cognition (Graham et al. 2002). VDAC 1 knockout rats are also useful as a model for inherited ALS; decreased VDAC activity caused by mutated SOD1 causes expedited disease progression and death at an earlier age than other ALS models (Israelson et al. 2010).

\section{Twinkle (or deletor) knockout mouse}

Twinkle, a nuclear-encoded mitochondrial DNA (mtDNA) helicase, may cause adult-onset progressive external ophthalmoplegia (PEO) with multiple mtDNA deletions (Tyynismaa et al. 2005). The Twinkle mouse model generated for these mutations shows the same symptomology as PEO patients and show progressive deficiencies in cytochrome c oxidase. However, these animals do not age prematurely, and do not show deficiencies in motor coordination. When these mice were tested in the rope grip, rotarod and wheel running paradigms, they showed no significant decreases in motor coordination, muscle strength or gaiting (Tyynismaa et al. 2005). Therefore, although these mice are a sufficient model for some aspects of adult-onset mitochondrial disorders, such as PEO, this particular model would be insufficient for testing in paradigms assessing cognition and motor function, as they showed no abnormalities in brain function or pathology.

\section{Behavioral modeling of mitochondrial dysfunction}

When studying the effects of any pharmacological or nutraceutical intervention on neurodegenerative and psychiatric disorders, it becomes necessary to observe the effects of that therapy on behavior. The above named mouse and rat in vivo models offer a unique opportunity to examine the effects of pharmaceutical and nutraceutical interventions on behaviors such as motor coordination, learning and memory, and nociception, for example (Crawley 1999). When using mouse models for mitochondrial dysfunction, it becomes particularly interesting to examine behaviors associated with motor coordination and cognition, as these areas of the brain may be the most affected by these dysfunctions. However, one must exercise caution when using animal models: the gender, age and genotype of the animal can play a pivotal role in the experimental outcome. For example, MPTP treatment results in significantly higher levels of neurotoxicity in older animals; younger animals seem better able to recover functionality after MPTP-induced damage (Patki et al. 2009). It is therefore crucial, when using the MPTP model of induction, to take the age of the experimental animals into account.

\section{Muscular coordination and motor function}

The rotarod and rope grip paradigms are tests for motor function and coordination, and are more commonly used for examining behaviors related to movement disorders (HD, PD, and ALS). The rotarod requires the animal to walk on a rotating rod which is slowly increasing in speed from 4 to approximately $40 \mathrm{rpm}$. The purpose of this paradigm is to examine motor coordination, and the gradual increase in rpm allows for animals that have no handicaps in motor function to move comfortably. The rope grip test, similar to the rotarod, requires the animal, when placed on a rope, to grasp the rope with both foreand hind limbs in order to shimmy to one end in order to escape. Those animals with decreased motor function and muscle strength will generally fall from the rotarod and the rope earlier than those without. However, when working with any genetically modified or induction model, it is important to perform these simple tests to rule out motor coordination dysfunctions before testing in other paradigms. Defects in motor coordination may lead to false negative results in other behavioral paradigms involving movement and behavior, such as cognition and anxiety tests, and should therefore be examined as early as possible before further testing takes place. 


\section{Cognition}

Mitochondrial-induced neuronal death in such brain areas as the hippocampus may result in cognitive deficits, as seen in patients with AD (Dragicevic et al. 2010). There are several paradigms that may be used to test cognition in rodents. Among them are the Morris water maze, the Y-maze, and the novel object recognition test. The Morris water maze gives a comprehensive overview of hippocampal memory, including spatial memory, long-term memory and long-term spatial memory, as well as learning abilities in general. During the extensive training phase, rodents are placed in a heated pool of water (1-1.5 meters in diameter, heated to about $\left.24^{\circ} \mathrm{C}\right)$, separated into four quadrants, containing a small $(5-10 \mathrm{~cm}$ in diameter) platform in one of the quadrants. The platform is usually hidden, but may sometimes be marked with a small flag. Each training trial consists of four swimming trials, in which the rodent is released at four different points in the maze, once in each quadrant. After the four trials, the animal is removed from the water, and after an inter-trial interval of anywhere between 60 to 120 minutes, will undergo another training session. All animals undergo two to four training sessions per day, usually for four consecutive days. Importantly, the platform placement remains the same for each animal (i.e., for some animals, the platform is always in quadrant 1 , for others, quadrant 2 , and so forth); visual cues are placed around the maze to assist the animal with orientation. The test, or probe trial, is performed on the fifth day of testing; the platform is removed from the maze, and the animal is allowed to search for 60 seconds. Time spent in the correct quadrant, and crosses over the platform location, give an indication of how well the animal has learned the task and its memory of the platform location. The probe test can also be done days and even weeks after the training trials, which is known as an extinction test, in order to observe the animals' long-term memory. Animals with learning deficits will spend equal amounts of time in all quadrants.

The Y-maze paradigm tests the working memory of rodents, by either testing their spontaneous alternation or spatial recognition. As the name implies, the maze itself is in the shape of a "Y", and it is possible to close one or two of the maze arms. However, to test spatial working memory, the spontaneous alternation test requires that all arms be open. The animals are placed at the end of the designated entry arm and allowed to explore for five minutes. The amounts of alternations are recorded and a score is calculated. Alternations are characterized as the following: assuming the entry arm is called A, and the other two arms are called $B$ and $C$, the animal should alternate between the arms in a consecutive $\mathrm{A}-\mathrm{B}-\mathrm{A}-\mathrm{C}$ or $\mathrm{A}-\mathrm{C}-\mathrm{A}-\mathrm{B}$ pattern. An arm entry is scored when the animal enters the arm with all four paws. The percent alternation can then be calculated as such: (number of alternations/ (total number of arm visits -2$)) * 100$. For example, if a mouse has a (total of 19 complete alternations/ $(27$ total arm visits -2$)) * 100=76 \%$ alternation rate; between $60 \%$ and $80 \%$ alternation is normal. Testing for spatial recognition memory requires that one arm of the Y-maze be blocked, and the animal allowed to explore the other two open arms. After a 5 to 15 minute exploration period, the animal is removed, and will be placed back in the maze, with the blocked arm now open, after an inter-trial period of 30 minutes to 24 hours. The time spent in the novel arm, the latency to exploring the novel arm, and numbers of entries into the novel arm give an indication of the animal's memory of the familiar, already explored territory and the novel area. Animals with severe memory deficits will not alternate and will spend equal amounts of time in the familiar and novel arms.

Similar to the Y-maze paradigm, novel object exploration can also be used to test an animal's memory of familiar and novel objects. In this paradigm, the animals are placed in an open field arena containing two identical objects. After an acclimation period of 5 to 15 minutes, the animals are removed from the open field and placed back in their home cage for an inter-trial interval of 30 minutes to 24 hours. For the test trial, one of the familiar objects is removed and replaced with an object that differs from the remaining familiar object. The animal is then placed back in the open field, and the time spent exploring the new object and the time spent in the area around it is recorded. Again, animals with memory deficits will not show any preference for the novel or familiar object. However, a word of warning about animal testing with novelty: rodents can also show aversions to novel situations and objects, and while their first instinct is usually to explore the unfamiliar, some animals will show an aversion to novelty and avoid the new object or environment altogether. It is therefore important to take this possible confounder into account when using tests focusing on novelty as a measure for memory.

\section{Depression, anxiety and schizophrenia}

The most popular models for depression are based on the concept of "learned helplessness" in rodents. These paradigms consist of a slightly noxious stimulus, such as being suspended by the tail or placement in a beaker of warm water, known respectively as the tail suspension and forced swim (or Porsolt swim test) paradigms. These paradigms both focus on the time spent by the animal in struggling/attempting to escape the situation. When the animal ceases to struggle, this is scored as "learned helplessness". Antidepressants have been found to be efficacious in both these paradigms, as animals receiving 
drug treatment struggle/swim longer than those receiving the vehicle.

Anxiety can best be observed in rodents using paradigms designed to examine behaviors in a mildly stressful environment, such as an open field or elevated plus maze. Both of these paradigms are used to examine anxiety behaviors in rodents; the elevated plus maze consists of a +- shaped maze, usually half a meter above the floor, containing two open and two closed arms. Animals showing less anxiety behaviors will enter and spend more time on the open (exposed) arm than those with more stress. Similarly, the open field paradigm, which consists of a large open arena, usually about 20 to $50 \mathrm{~cm}$ in diameter, allows the observer to examine how often a rodent will enter the center, more stressful, area of the open field, as opposed to the less stressful periphery.

The best known paradigm for schizophrenia testing is known as pre-pulse inhibition of startle. This paradigm, as the name implies, examines the startle capabilities of the test subjects in response to a loud auditory stimulus of $120 \mathrm{~dB}$. Inhibition of startle behavior is achieved by a short pre-pulse, milliseconds before the startle stimulus, of either 80,82 or $85 \mathrm{~dB}$. In normal rodents, the pre-pulse will decrease the startle amplitude, but in models showing schizophrenic behavior, no inhibition of startle is apparent.

\section{Pharmaceutical and nutraceutical interventions for mitochondrial disorders}

The above named animal models and behavioral paradigms can be used to explore the efficacy of known and novel treatments for diseases resulting from mitochondrial dysfunction (Table 3). The following is a brief overview of the most used pharmaceutical and nutraceutical interventions for mitochondrial dysfunction-induced neurodegenerative diseases and their effects on the previously mentioned behaviors (for more comprehensive reviews, please see: Liu et al. 2002; Navarro and Boveris 2008; Frisar and Hroudova 2010).

\section{Antidepressants}

Mitochondrial dysfunction has long been known to be tied to the development of several neurodegenerative diseases, among which are PD, HD, AD and ALS (Lin and Beal 2006). These deficiencies have also been related to the development of depression, anxiety, and schizophrenia (Scaglia 2010). Along these lines, several forms of antidepressants have been examined for their putative effects on monoamine systems in the previously mentioned diseases. Several kinds of dopamine agonists have been examined in the treatment of $\mathrm{PD}$, including pramipexole and D-264, both dopamine $\mathrm{D}_{3}$ receptor agonists. Both of these treatments have been shown to improve motor performance on the rotarod and rope grip tests in the MPTP induction model of PD ( $\mathrm{Li}$ et al. 2010), and interestingly, D-264 also increases brain-derived neurotrophic factor (BDNF) levels in MPTP-treated mice, suggesting that BDNF may also play a role in neuroprotection (Li et al. 2010; $\mathrm{Wu}$ et al. 2010). At the mitochondrial level, these compounds are thought to be neuroprotective, blocking the effects of MPTP on mitochondrial complex I inhibition and dopaminergic neuron loss ( $\mathrm{Li}$ et al. 2010).

Similarly, the tricyclic antidepressant (TCA) nortriptyline delayed disease onset and mortality in $S O D 1^{G 93 A}$ mice, and rescued motor neurons in the spinal cord. The authors hypothesize that treatment with this drug inhibited mitochondrial cytochrome $\mathrm{c}$ release and caspase activation in the treated animals, protecting against ALS-associated neurodegeneration. Treatment also delayed disease onset in R6/2 knockout mice and delayed onset of rotarod-related motor deficiencies, but accelerated disease progression once symptoms began to manifest (Wang et al. 2007). The authors suggest that this accelerated disease progression is the result of accumulated toxicity of nortripyline after five months of administration. These results suggest that nortriptyline treatment, if administered early enough, may be able to circumvent the development of ALS, perhaps as well as other neurodegenerative diseases (Wang et al. 2007), but would not be a useful treatment for those already suffering from such afflictions.

The mood stabilizer lithium has been shown to be efficacious in the SOD1 ${ }^{G 93 A}$ transgenic mouse model of ALS; animals receiving daily doses of lithium had an increased survival time of $36 \%$ (Fornai et al. 2008). Treatment also delayed the onset of paralysis and limb adduction, and improved performance in the rotarod and grip strength paradigms (Fornai et al. 2008). Animals began receiving treatment at day 75 , when they were already quite old, but not yet showing symptoms of the disease. The authors hypothesize that treatment with lithium was neuroprotective, in that it rescued spinal cord mitochondria and produced significant decreases in $\alpha$-synuclein, ubiquitin, and SOD1 aggregates. To date, it is unclear whether treatment with lithium after symptoms have begun is efficacious in reversing damage to mitochondria in motor neurons.

Serotonin receptor expression has been shown to be decreased in the R6/1 mouse model for HD, and this mouse also shows symptomology similar to those suffering from depression and HD (Pang et al. 2009). Treatment with the SSRI sertraline improved performance in the forced swim depression paradigm: female knock-ins receiving sertraline showed less immobility time compared to controls, but there was no effect on male behavior. Interestingly, male 


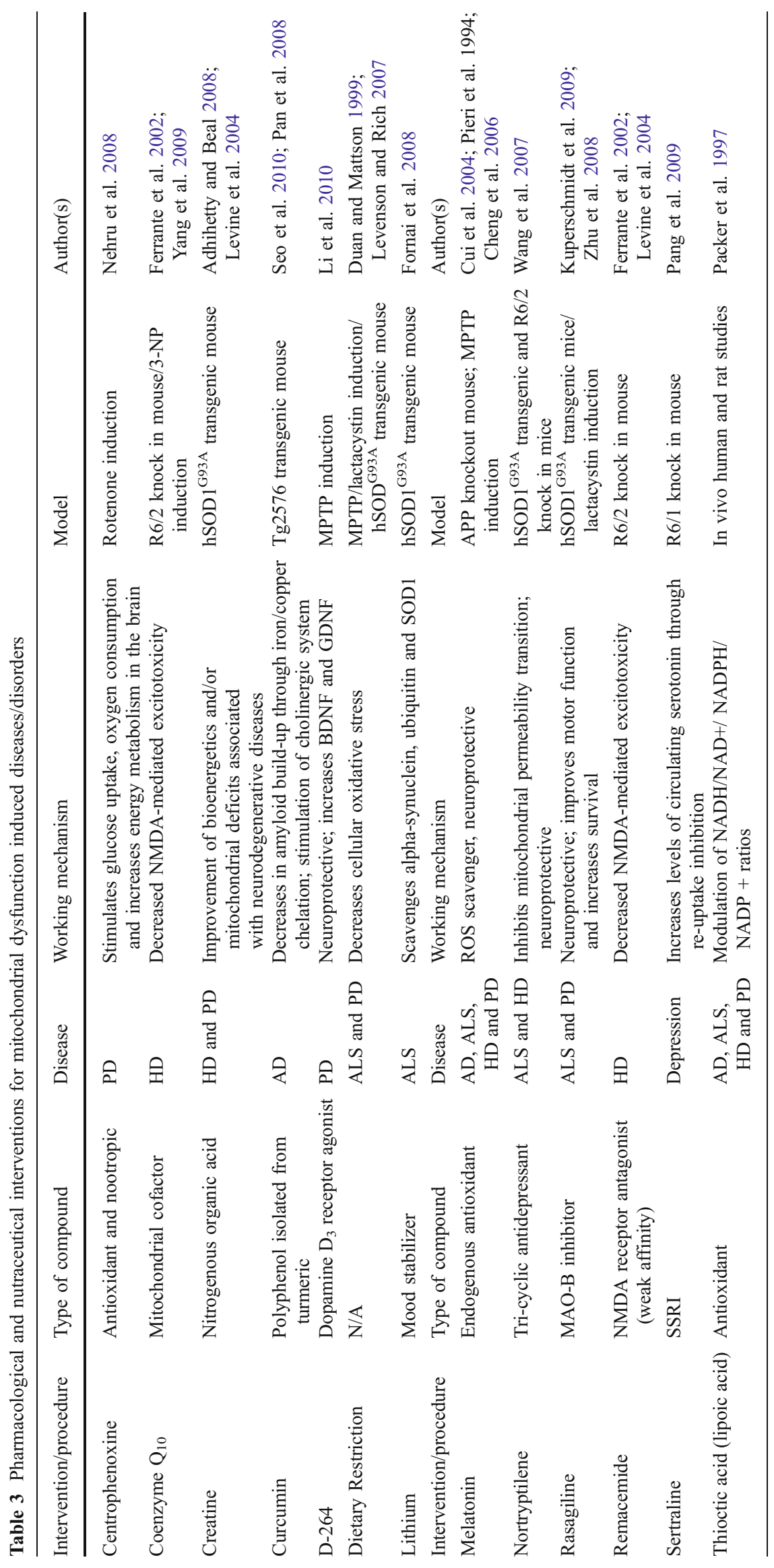


knock-ins showed improvements in the tail suspension paradigm when treated with either sertraline or desipramine (a TCA), but there was no effect of either drug on female performance in the tail suspension paradigm. Further, environmental enrichment also decreased depressionrelated behaviors in the forced swim test in female knockouts, but had no effect on males (Pang et al. 2009). However, the working mechanisms of this SSRI on mitochondrial function remain unknown; Kumar et al. (2011) theorize that treatment with compounds like sertraline may protect against oxidative damage and subsequent neuronal death from nitrogenous free radicals.

\section{Antioxidants}

Since mitochondrial damage is highly attributed to oxidative damage from ROS production (among others), the examination of antioxidants in the battle against neurodegenerative and psychiatric disorders has become a topic of much examination over the past years. In a study examining the effects of lipoic acid (an organosulfur compound and putative antioxidant found in almost all food, but in higher concentrations in spinach, broccoli and liver) Packer et al. (1997) found that treatment with this compound resulted in improved motor performance and cognition in older mice (20-23 months old), while there were no effects in younger animals. Other experiments with lipoic acid have shown that this antioxidant also increases locomotion as well as improving memory in older rats (Liu et al. 2002), which indicates that this particular compound may be efficacious in the treatment of AD. These particular experiments have many advantages, as they examined the effects of lipoic acid in older mice without using any pharmacological induction, a situation much more similar to that of human patients.

Examinations of L-carnitine and its acetyl derivative, acetyl L-carnitine (ALCAR), which both provide the acetyl equivalents for the production of the neurotransmitter acetylcholine, have shown that both of these antioxidants result in improvements in age associated declines in cognition and locomotion, making these nutraceuticals suitable for further use in AD research (Liu et al. 2002). However, ALCAR is more often used in experimentation, as it is better absorbed and passes more easily through the blood brain barrier than L-carnitine. ALCAR also protects neurons from toxicity from those compounds functioning as mitochondrial uncouplers or inhibitors (Virmani et al. 1995), and increases brain GABA levels (Liu et al. 2002). Interestingly, this antioxidant seems to have better results at lower doses; when given at concentrations of $0.15 \%$, $0.2 \%$ and $0.5 \%$, treatment resulted in decreases in mitochondrial loss in the hippocampal dentate gyrus of older animals (Liu et al. 2002). Treatment with this compound at $1.5 \%$ did not have as drastic effects as the lower doses, perhaps either due to a saturation effect or perhaps this antioxidant becomes neurotoxic at higher doses. Studies examining the combined effects of ALCAR with lipoic acid show significant increases in improvements on spatial memory, compared to treatment with either antioxidant alone (Liu et al. 2002).

Oral administration of the antioxidant creatine (a nitrogenous organic acid) resulted in motor performance improvements, extended life span, and protected against neuronal loss in the substantia nigra and motor cortex of $S O D 1^{G 93 A}$ mice, indicating that this organic acid may be useful in future studies examining the effects of creatine on such movement disorders as PD and ALS. Treatment with this compound also decreased the number of Huntington positive aggregates in the brain, but was not efficacious in alleviating deficits in cognition (Adhihetty and Beal 2008), rendering this particular antioxidant less suitable for the treatment for the cognitive deficits associated with $\mathrm{AD}$.

Melatonin is an endogenous antioxidant manufactured by the pineal gland and slows the process of neuronal senescence (Srinivasan et al. 2006). Four months' administration of melatonin to amyloid precursor protein (APP) transgenic mice, an in vivo AD model, resulted in significant improvements in cognition, learning and memory, and decreased $A \beta$ deposits in the frontal cortex (Cheng et al. 2006). The authors also show that melatonin administration decreases oxidative stress and neuronal apoptosis, perhaps through increases in SOD activity and inhibition of lipid peroxidase production (Cheng et al. 2006).

Centrophenoxine, an antioxidant and nootropic, improves motor function on the rotarod and has positive effects on DA levels after rotenone treatment, a popular induction model of PD (Nehru et al. 2008). Studies with this compound have also found that it can improve cognitive performance in rats injected with the muscarinic antagonist scopolamine, used to impair memory (Petkov et al. 1988). Centrophenoxine is thought to work by stimulating glucose uptake and oxygen consumption and increasing energy metabolism in the brain. However, this nootropic has been little examined in other animal models for neurodegenerative diseases, and may prove effective in models for $\mathrm{AD}$ as well.

\section{Other pharmaceutical and nutraceutical interventions}

The monoamine oxidase-B (MAO-B) inhibitors rasagiline and seleginine show promising results in $\mathrm{C} 57 \mathrm{Bl} / 6$ mice with a compromised ubiquitin-proteasome (UPS) system, achieved through microinjection of the drug lactacystin, a UPS inhibitor, in the median forebrain bundle (Zhu et al. 2008). The resulting phenotype is similar to that of PD patients; pre-treatment with these two compounds not only 
improves motor performance in these mice, but also protects dopamine neurons from lactacystin-induced damage and increased the concentrations of dopamine and its metabolites 3,4-dihydroxy-phenylacetic acid (DOPAC) and homovanillic acid (HVA) (Zhu et al. 2008). However, treatment after lactacystin-induced damage did not result in such robust improvements: there were no significant effects on rotarod performance, but some improvement in open field motility (Zhu et al. 2008).

$\mathrm{M} 30$, an iron chelating compound derived partly from rasagiline, decreases motor dysfunctions and prolongs the lifespan of elderly $S O D 1^{G 93 A}$ mice (Kuperschmidt et al. 2009). The authors hypothesized that this compound may work by regenerating motor nerves, inducing neurodifferentiation and axon sprouting, all leading to the re-innervation of muscle fibers. Interestingly, treatment with M30 also raises BDNF levels, suggesting a greater role for this neurotrophic factor than previously believed. Many have hypothesized that BDNF plays a major role in the development of depression (Krishnan and Nestler 2010; Cryan and Slattery 2010), and therefore may be a key component in many cases of depression comorbidity seen in those with neurodegenerative diseases, because neurodegeneration may result in decreased BDNF levels, neuronal damage in the hippocampus, and subsequent depression.

Pre-treatment with the non-metabolizable glucose analogue 2 deoxyglucose (2DG), combined with dietary restriction, reduced damage to dopamine neurons in the substantia nigra and improved motor behaviors on the rotarod in mice exposed to MPTP, an induction model for PD (Duan and Mattson 1999). The authors theorize that 2DG works by reducing basal levels oxidative stress though decreasing protein oxidation and that, combined with dietary restriction, results in a "stress response" which may have a beneficial effect on lifespan prolongation. Previous studies have shown that caloric restriction can indeed have beneficial effects on neurodegenerative diseases, perhaps by influencing adult neurogenesis (Levenson and Rich 2007). However, extensive research needs to be done before this can be proven equivocally. This experiment does not show the effects of either 2DG or dietary restriction on animals already exposed to MPTP, and whether or not dietary restriction and/or 2DG exposure would help to repair damage already present in the brain.

Coenzyme $\mathrm{Q}_{10}$, a component of the electron transport chain and a key constituent in cellular respiration, given in conjunction with the NMDA receptor antagonist remacimide, results in increased survival time in a transgenic model for HD, R6/2 mice. Combined treatment was more efficacious than treatment with either compound by itself; mice treated with this combination showed significant improvements in rotarod performance and less loss of body weight compared to those groups treated with either compound by itself (Ferrante et al. 2002). Further, coenzyme $\mathrm{Q}_{10}$ has been shown to be neuroprotective in 3NP induction models (Yang et al. 2009). These results further underline the point that treatment for mitochondrial dysfunction diseases will not be straightforward; there will not be one "cure-all" for the disease. Multiple combinations of treatments may be necessary not only to combat the symptoms of these diseases, but to cure them as well.

Treatment with the spice curcumin, an active polyphenol isolated from turmeric (Curcuma longa), has been shown to improve cognitive deficits in the Tg2576 AD mouse model (Seo et al. 2010). Curcumin exposure not only rectified gaiting deficiencies seen in the transgenic mice, it also resulted in significant decreases in characteristic plaque pathology and neuronal loss in the brain, similar to what is seen in human AD patients (Seo et al. 2010). Others have also shown that curcumin also improves performance in the Morris water maze; mice receiving curcumin showed shorter escape latency compared to vehicles (Kumar et al. 2011). Some hypothesize that this compound acts to reduce oxidative damage, plaque build-up and levels of insoluble amyloid, possibly through chelating iron and copper on beta amyloid, and can improve cognition through the stimulation of the cholinergic system (Pan et al. 2008).

\section{Conclusions}

The literature reviewed above gives a large overview of previous studies performed with animal models of mitochondrial dysfunction, and the effects of pharma- and nutraceutical interventions on behavior. However, it is of note that almost all of these studies focus on the neuroprotective qualities of the given compound. There is little data on the power of these treatments to cure or at least treat symptoms once they have begun to manifest. While neuroprotection is an important aspect of preventing neurodegenerative and CNS afflictions, it is not reasonable to suggest that the population at large begin chronic treatment with antidepressants, for example. The ideal solution would be to eliminate the disease through gene therapy, removing the cause of the disease at its source, before symptoms even begin. However, this is assuming that all diseases have a genetic basis, which may not be entirely true. It is of note that for some models of mitochondrial dysfunction, there is no need for genetic manipulation or pharmacological induction. Studies done by Gong et al. (2010) have shown that chronic stress may result in damages to mitochondrial ultrastructure and function in the brain. It has already been hypothesized by many that dysfunctions in the hypothalamus-pituitaryadrenal gland (HPA) axis, due to increased levels of stress 
hormones, may also result in stress and depression, both of which have been linked to mitochondrial dysfunctions (Braw et al. 2006; Garcia et al. 2011; Clay et al. 2010). It may therefore be so that, in conjunction with genetic modification and induction models, environmental models like those of chronic stress could also be used to study the effects of mitochondrial dysfunctions on brain disorders. The causes of some diseases are as of yet still unknown, but factors such as environment and diet may also play key roles in disease development. We need to be able to find the source of these dysfunctions and prevent them from leading to the aforementioned maladies, and herein lays our greatest challenge.

The glut of studies done examining mitochondrial dysfunction diseases shows us that there is still much to be learned about the nature of these illnesses, their causes, and their cures. With the help of animal models, pharmaceutical and nutraceutical interventions, we can perhaps make further progress into (and hopefully out of) the mire. However, it is imperative to remember that the answer to this riddle may be complicated; it may be that no one treatment will be the "magic bullet"; the answer may lie in a combination of nutra- and pharmaceuticals, perhaps in conjunction with external environmental influences, such as dietary restriction. While current animal models are certainly imperfect and may not necessarily mirror all the symptoms and pathology of human patients, they are invaluable for examining specific aspects of mitochondrial dysfunctions, and should be recognized for the advantages they offer to the study of neurodegenerative diseases.

Open Access This article is distributed under the terms of the Creative Commons Attribution Noncommercial License which permits any noncommercial use, distribution, and reproduction in any medium, provided the original author(s) and source are credited.

\section{References}

Adhihetty PJ, Beal MF (2008) Creatine and its potential therapeutic value for targeting cellular energy impairment in neurodegenerative diseases. Neuromolecular Med 10(4):275-290

Alam M, Mayerhofer A, Schmidt WJ (2004) The neurobehavioral changes induced by bilateral rotenone lesion in medial forebrain bundle of rats are reversed by L-DOPA. Behav Brain Res 15(1-2):117-124

Andres-Mateos E, Perier C, Shang L et al. (2007) DJ-1 gene deletion reveals that DJ-1 is an atypical peroxiredoxin-like peroxidase. Proc Natl Acad Sci USA 104:14807-14812

Andrews ZB, Diano S, Horvath TL (2005) Mitochondrial uncoupling proteins in the CNS: in support of function and survival. Nat Rev Neurosci 6(11):829-840

Attems J, Ittner A, Jellinger K, Nitsch RM, Maj M, Wagner L, Gotz J, Heikenwalder M (2011) Reduced secretagogin expression in the hippocampus of P301L tau transgenic mice. J Neural Transm 118 (5):737-745
Benit P, Goncalves S, Dassa EP, Briere JJ, Rustin P (2008) The variability of the harlequin mouse phenotype resembles that of human mitochondrial-complex I-deficiency syndromes. PLos One 3(9):e3208

Bennett MC, Mlady GW, Kwon YH, Rose GM (1996) Chronic in vivo sodium azide infusion induces selective and stable inhibition of cytochrome c oxidase. J Neurochem 66(6):2606-2611

Ben-Shachar D (2009) The interplay between mitochondrial complex I, dopamine and Sp1 in schizophrenia. J Neural Transm 116:1383-1396

Boveris A, Navarro A (2008) Brain mitochondrial dysfunction in aging. IUBMB Life 60(5):308-314

Braw Y, Malkesman O, Merlender A et al. (2006) Stress hormones and emotion-regulation in two genetic animal models of depression. Psychoneuroendocrinology 31(9):1105-1116

Browne SE, Bowling AC, MacGarvey U et al. (1997) Oxidative damage and metabolic dysfunction in Huntington's disease: selective vulnerability of the basal ganglia. Ann Neurol 41 (5):646-653

Carreras MC, Franco MC, Finocchietto PV et al. (2007) The biological significance of mtNOS production. Front Biosci 12:1041-1048

Chen CM (2011) Mitochondrial dysfunction, metabolic deficits, and increased oxidative stress in Huntington's disease. Chang Gung Med J 34(2):135-152

Cheng Y, Feng Z, Zhang Q, Zhang J (2006) Beneficial effects of melatonin in experimental models of Alzheimer's disease. Acta Pharm Sinica 27(2):129-139

Choi WS, Kruse SE, Palmiter RD, Xia Z (2008) Mitochondrial complex I inhibition is not required for dopaminergic neuron death induced by rotenone, MPP + or paraquat. Proc Natl Acad Sci 105(39):15136-15141

Chou AP, Li S, Fitzmaurice AG, Bronstein JM (2010) Mechanisms of rotenone-induced proteasome inhibition. Neurotoxicology 31 (4):367-372

Clay HB, Sillivan S, Konradi C (2010) Mitochondrial dysfunction and pathology in bipolar disorder and schizophrenia. Int $\mathrm{J}$ Dev Neurosci. doi:10.1016/j.ijdevneu.2010.08.007

Crawley JN (1999) Behavioral phenotyping of transgenic and knockout mice: experimental design and evaluation of general health, sensory functions, motor abilities, and specific behavioral tests. Brain Res 835(1):18-26

Cryan JF, Slattery DA (2010) GABAB receptors and depression. Current status. Adv Pharmacol 58:427-451

Cui K, Luo X, Xu K, Ven Murthy MR (2004) Role of oxidative stress in neurodegeneration: recent developments in assay methods for oxidative stress and nutraceutical antioxidants. Prog Neuropsychopharmacol Biol Psych 28:771-799

Dawson TM, Ko HS, Dawson VL (2010) Genetic animal models of Parkinson's disease. Neuron 66(5):646-661

de Moura MB, dos Santos LS, Van Houten B (2010) Mitochondrial dysfunction in neurodegenerative diseases and cancer. Environ Mol Mutagen 51(5):391-405

Dragicevic N, Mamacarz M, Zhu Y, Buzzeo R, Tan J, Arendash GW, Bradshaw PC (2010) Mitochondrial amyloid-beta levels are associated with the extent of mitochondrial dysfunction in different brain regions and the degree of cognitive impairment in Alzheimer's transgenic mice. J Alzheimer's Dis 20(Suppl 2): S535-S550

Duan W, Mattson MP (1999) Dietary restriction and 2-Deoxyglucose administration improve behavioral outcome and reduce degeneration of dopaminergic neurons in models of Parkinson's disease. J Neurosci Res 57:195-206

Duan W, Guo Z, Mattson MP (2000) Participation of par-4 in the degeneration of striatal neurons induced by metabolic compromise with 3-nitropropionic acid. Exp Neurol 165 (1): $1-11$ 
Ferrante RJ, Andreassen OA, Dedeoglu A, Ferrante KL, Jenkins BG, Hersch SM, Beal MF (2002) Therapeutic effects of coenzyme $\mathrm{Q}_{10}$ and remacemide in transgenic mouse models of Huntington's disease. J Neurosci 22(5):1592-1599

Fornai F, Longone P, Cafaro L et al. (2008) Lithium delays progression of amyotrophic lateral sclerosis. PNAS 105(6):2052-2057

Frisar Z, Hroudova J (2010) Intracellular signaling pathways and mood disorders. Folia Biol (Praha) 56(4):135-148

Galter D, Pernold K, Yoshitake T et al. (2010) MitoPark mice mirror the slow progression of key symptoms and L-DOPA response in Parkinson's disease. Genes Brain Behav 9(2):173-181

Garcia FD, Coquerel Q, Kijve E, Dechelotte P, Harro J, Fetissoy SO (2011) Autoantibodies reacting with vasopressin and oxytocin in relation to cortisol secretion in mild and moderate depression. Prog Neuropsychopharmacol Biol Psychiatry 35 (1):118-125

Gardner A, Boles RG (2010) Beyond the serotonin hypothesis: Mitochondria, inflammation and neurodegeneration in major depression and affective spectrum disorders. Prog Neuropsychopharmacol Biol Psychiatry. doi:10.1016/j.pnpbp.2010.07.030

Gasser T (2009) Molecular pathogenesis of Parkinson disease: insights from genetic studies. Expert Rev Mol Med 11:e22

Ghezzi D, Sevrioukova I, Invernizzi F et al. (2010) Severe X-linked mitochondrial encephalomyopathy associated with a mutation in apoptosis-induced factor. Am J Hum Genet 86(4):639-649

Gong Y, Chai Y, Ding JH, Sun XL, Hu G (2010) Chronic mild stress damages mitochondrial ultrastructure and function in mouse brain. Neurosci Lett. doi:10.1016/j.neulet.2010.11.006

Goodall EF, Morrison KE (2006) Amyotrophic lateral sclerosis (motor neuron disease): proposed mechanisms and pathways to treatment. Expert Rev Mol Med 8(11):1-22

Graham BH, David Sweatt J, Craigen WJ (2002) Noninvasive, in vivo approaches to evaluating behavior and exercise physiology in mouse models of mitochondrial disease. Methods 26(4):364-370

Green DR, Kroemer G (2004) The pathophysiology of mitochondrial cell death. Science 305(5684):626-629

Gurney ME, Pu H, Chiu AY et al. (1994) Motor neuron degeneration in mice that express a human $\mathrm{Cu}, \mathrm{Zn}$ superoxide dismutase mutation. Science 264(5166):1772-1775

Hanrott K, Gudmunsen L, O'Neill MJ, Wonnacott S (2006) 6Hydroxydopamine-induced apoptosis is mediated via extracellular auto-oxidation and caspase-3 dependent activation of protein kinase C8*. J Biol Chem 281(9):5373-5382

Hirai K, Aliev G, Nunomura A et al. (2001) Mitochondrial abnormalities in Alzheimer's disease. J Neurosi 21(9):3017-3023

Ingraham CA, Burwell LS, Skalska J, Brookes PS, Howell RL, Sheu SS, Pinkert CA (2009) NDUFS4: creation of a mouse model mimicking a Complex I disorder. Mitochondrion 9(3):204-210

Ishimura R, Martin GR, Ackerman SL (2008) Loss of apoptosisinducing factor results in cell-type-specific neurogenesis defects. J Neurosci 28(19):4938-4948

Israelson A, Arbel N, Da Cruz S, Ilieva H, Yamanaka K, ShoshanBarmatz V, Cleveland DW (2010) Misfolded mutant SOD1 directly inhibits VDAC1 conductance in a mouse model of inherited ALS. Neuron 67(4):575-587

Janus C, D'Amelio S, Amitay O et al. (2000) Spatial learning in transgenic mice expressing human presenilin 1 (PS1) transgenes. Neurobiol Aging 21(4):541-549

Kim GW, Copin JC, Kawase M, Chen SF, Sato S, Gobbel GT, Chan $\mathrm{PH}$ (2000) Excitotoxicity is required for induction of oxidative stress and apoptosis in mouse striatum by the mitochondrial toxin, 3-nitroproprionic acid. J Cereb Blood flow Metab 20 (1):119-129

King DL, Arendash GW (2002) Behavioral characterization of the Tg2576 transgenic model of Alzheimer's disease through 19 months. Physiol Behav 75(5):627-642
Krishnan V, Nestler EJ (2010) Linking molecules to mood: new insight into the biology of depression. Am J Psychiatry 167 (11): $1305-1320$

Kruse SE, Watt WC, Marcinek DJ, Kapur RP, Schenkman KA, Palmiter RD (2008) Mice with mitochondrial complex I deficiency develop a fatal encephalomyopathy. Cell Metab 7(4):312-320

Kulic L, Wollmer MA, Rhein V et al. (2009) Combined expression of tau and the Harlequin mouse mutation leads to increased mitochondrial dysfunction, tau pathology and neurodegeneration. Neurobiol Aging. doi:10.1016/j.neurobiolaging.2009.10.014

Kumar A, Prakash A, Dogra S (2011) Protective effect of curcumin (Curcuma longa) against D-galactose-induced senescence in mice. J Asian Nat Prod Res 13(1):42-55

Kuperschmidt L, Weinreb O, Amit T, Mandel S, Carri MT, Youdim MBH (2009) Neuroprotective and neuritogenic activities of novel multimodal iron-chelating drugs in motor-neuron-like NSC-34 cells and transgenic mouse model of amyotrophic lateral sclerosis. FASEB J 23:3766-3779

Langston JW, Ballard P, Tetrud JW, Irwin I (1983) Chronic Parkinsonism in humans due to a product of meperidine-analog synthesis. Science 219(4587):979-980

Lenzken SC, Romeo V, Zolezzi F et al. (2011) Mutant SOD1 and mitochondrial damage alter expression and splicing of genes controlling neuritogenesis in models of neurodegeneration. Hum Mutat 32(2): 168-182

Levenson CW, Rich NJ (2007) Eat less, live longer? New insights into the role of caloric restriction in the brain. Nutr Rev 65 (9):412-415

Levine MS, Klapstein GJ, Koppel A et al. (1999) Enhanced sensitivity to N-methyl-D-aspartate receptor activation in transgenic and knockin mouse models of Huntington's disease. J Neurosci Res 58(4):515-532

Levine JS, Cepeda C, Hickey MA, Fleming SM, Chesselet MF (2004) Genetic mouse models of Huntington's and Parkinson's diseases: illuminating but imperfect. Trend Neurosci 27 (11):691-697

Li C, Biswas S, Li X, Dutta AK, Le W (2010) Novel D3 dopamine receptor-preferring agonist D-264: Evidence of neuroprotective property in Parkinson's disease animal models induced by 1methyl-4-phenyl-1,2,3,6-tetrahydropyridine and lactacystin. J Neurosci Res 88:2513-2523

Lin MT, Beal MF (2006) Mitochondrial dysfunction and oxidative stress in neurodegenerative diseases. Nature 443(7113):787-795

Liu J, Atamna H, Kuratsune H, Ames BN (2002) Delaying brain mitochondrial decay and aging with mitochondrial antioxidants and metabolites. Ann NY Acad Sci 959:133-166

Masliah E, Mallory M, Hansen L, DeTeresa R, Alford M, Terry R (1994) Synaptic and neuritic alterations during the progression of Alzheimer's disease. Neurosci Lett 174(1):67-72

Mounsey RB, Teismann P (2010) Mitochondrial dysfunction in Parkinson's disease: pathogenesis and neuroprotection. Parkinson's Dis 2011:617472

Navarro A, Boveris A (2008) Mitochondrial nitric oxide synthase, mitochondrial brain dysfunction in aging, and mitochondriatargeted antioxidants. Adv Drug Deliv Rev 60(13-14):1534-1544

Nehru B, Verma R, Khanna P, Sharma SK (2008) Behavioral alterations in rotenone model of Parkinson's disease: attenuation by co-treatment of centrophenoxine. Brain Res 1201:122-127

Novak MJ, Tabrizi SJ (2010) Huntington's disease. BMJ 340:c3109. doi:10.1136/bmj.c3109

Oliveira JM (2010) Nature and cause of mitochondrial dysfunction in Huntington's disease: focusing on huntingtin and the striatum. J Neurochem 114(1):1-12

Packer L, Tritschler HJ, Wessel K (1997) Neuroprotection by the metabolic antioxidant $\alpha$-lipoic acid. Free Radical Bio Med 22:359-378 
Pan R, Qiu S, Lu DX, Dong J (2008) Curcumin improves learning and memory ability and its neuroprotective mechanism in mice. Chin Med J 121(9):832-839

Pang TYC, Du X, Zajac MS, Howard ML, Hannan AJ (2009) Altered serotonin receptor expression is associated with depressionrelated behavior in the R6/1 transgenic mouse model of Huntington's disease. Hum Mol Gen 18(4):753-766

Patki G, Che Y, Lau YS (2009) Mitochondrial dysfunction in the striatum of aged chronic mouse model of Parkinson's disease. Front Aging Neurosci 11:1-3

Peng Y, Sun J, Hon S, Nylander AN, Xia W, Feng Y, Want X (2010) L3 - $n$-Butylphthalide improves cognitive impairment and reduces amyloid- $\beta$ in a transgenic model of Alzheimer's disease. J Neurosci 30(24):8180-8189

Petkov VD, Mosharrof AH, Petkov VV (1988) Comparative studies on the effects of the nootropic drugs adafenoxate, meclofenoxate and piracetam, and of citicholine on scopolamine-impaired memory, exploratory behavior and physical capabilities (experiments on rats and mice). Acta Physiol Pharmacol Bulg 14(1):3-13

Pieri C, Marra M, Moroni F, Recchioni R, Marcheselli F (1994) Melatonin: a peroxyl radical scavenger more effective than vitamin E. Life Sci 55(15): PL271-276

Quintana A, Kruse SE, Kapur RP, Sanz E, Palmiter RD (2010) Complex I deficiency due to loss of Ndufs 4 in the brain results in progressive encephalopathy resembling Leigh syndrome. Proc Natl Acad Sci USA 107(24):10996-11001

Resende R, Moreira PI, Proenca T, Deshpande A, Busciglio J, Pereira C, Oliveira CR (2008) Brain oxidative stress in a tripletransgenic mouse model of Alzheimer's disease. Free Radic Biol Med 44:2051-2057

Reyes NA, Fisher JK, Austgen K, VandenBerg S, Huang EJ, Oakes SA (2010) Blocking the mitochondrial apoptotic pathway preserves motor neuron viability and function in a mouse model of amyotrophic lateral sclerosis. J Clin Invest 120(10):3673-3679

Scaglia F (2010) The role of mitochondrial dysfunction in psychiatric disease. Dev Disabil Res Rev 16(2):136-143

Schapira AH (2010) Complex I: inhibitors, inhibition and neurodegeneration. Exp Neurol 224(2):331-335

Schiff M, Benit P, Coulibaly A, Loublier S, El-Khoury R, Rustin P (2011) Mitochondrial response to controlled nutrition in health and disease. Nutr Rev 69(2):65-75

Searcy DG (2003) Metabolic integration during the evolutionary origin of mitochondria. Cell Res 13(4):229-238

Seo JS, Leem YH, Lee KW, Kim SW, Lee JK, Han PL (2010) Severe motor neuron degeneration in the spinal cord of the TG2576 mouse model of Alzheimer disease. J Alzheimers Dis 21(1):263-276

Shan X, Chiang PM, Prince DL, Wong PC (2010) Altered distributions of Gemini of coiled bodies and mitochondria in motor neurons of TDP-43 transgenic mice. Proc Natl Acad Sci USA 107 (37):16325-16330

Smeitink J, van den Heuvel L, DiMauro S (2001) The genetics and pathology of oxidative phosphorylation. Nat Rev Genet 2 (5):342-352

Srinivasan V, Pandi-Perumal SR, Cardinali DP, Poeggler B, Hardeland R (2006) Melatonin in Alzheimer's disease and other neurodegenerative disorders. Behav Brain Function 2:15-37

Strazielle C, Jazi R, Verdier Y, Qian S, Lalonde R (2009) Regional brain metabolism with cytochrome c oxidase histochemistry in a
PS1/A246E mouse model of autosomal dominant Alzheimer's disease: correlations with behavior and oxidative stress. Neurochem Int 55:806-814

Tang TS, Chen X, Liu J, Bezprozyanny I (2007) Dopaminergic signaling and striatal neurodegeneration in Huntington's disease. J Neurosci 27(30):7899-7910

Tansey MG, Goldberg MS (2010) Neuroinflammation in Parkinson's disease: its role in neuronal death and implications for therapeutic intervention. Neurobiol Dis 37(3):510-518

Tyynismaa H, Mjosund KP, Wanrooij S et al. (2005) Mutant mitochondrial helicase Twinkle causes multiple mtDNA deletions and a late-onset mitochondrial disease in mice. Proc Natl Acad Sci 102(49):17687-17692

Vali S, Mythri RB, Jagatha B et al. (2007) Integrating glutathione metabolism and mitochondrial dysfunction with implications for Parkinson's disease: a dynamic model. Neuroscience 149(4):917-930

Van Raamsdonk JM, Warby SC, Hayden MR (2007) Selective degeneration in YAC mouse models of Huntington disease. Brain Res Bull 72(2-3):124-131

Virmani MA, Biselli R, Spadoni A et al. (1995) Protective actions of L-carnitine and acetyl-L-carnitine on the neurotoxicity evoked by mitochondrial uncoupling or inhibitors. Pharmacol Res 32 (6):383-389

Wang LH, Qin ZH (2006) Animal models of Huntington's disease: implications in uncovering pathogenic mechanisms and developing therapies. Acta Pharmacol Sin 27(10):1287-1302

Wang J, Xiong S, Xie C, Markesbery WR, Lovell MA (2005) Increased oxidative damage in nuclear and mitochondrial DNA in Alzheimer's disease. J Neurochem 93:953-962

Wang H, Guan Y, Wang X et al. (2007) Nortriptyline delays disease onset in models of chronic neurodegeneration. Eur J Neurosci 26 (3):633-641

Weeber EJ, Levy M, Sampson MJ et al. (2002) The role of mitochondrial porins and the permeability transition pore in learning and synaptic plasticity. J Biol Chem 277(21):18891-18897

Weinstock M, Shoham S (2003) Rat models of dementia based on reductions in regional glucose metabolism, cerebral blood flow and cytochrome oxidase activity. J Neural Transm 111(3):347-366

Wu CL, Hwang CS, Chen SD, Yin JH, Yang DI (2010) Neuroprotective mechanisms of brain-derived neurotrophic factor against 3-nitropropionic acid toxicity; therapeutic implications for Huntington's disease. Ann N Y Acad Sci 1201:8-12

Xiong N, Huang J, Zhang Z et al. (2009) Stereotaxical infusion of rotenone: a reliable rodent model for Parkinson's disease. PLoS One 4(11):e7878

$\mathrm{Xu}$ YF, Gendron TF, Zhang YJ et al. (2010) Wild-type human TDP-43 expression causes TDP-43 phosphorylation, mitochondrial aggregation, motor deficits, and early mortality in transgenic mice. J Neurosci 30(32):10851-10859

Yang L, Calingasan NY, Wille EJ, Cormier K, Smith K, Ferrente RJ, Beal MF (2009) Combination therapy with conenzyme Q10 and creatine produces additive neuroprotective effects in models of Parkinson's and Huntington's diseases. J Neurochem 109 (5):1427-1439

Zhu W, Xie W, Pan T, Jankovic J, Li J, Youdim MBH, Le W (2008) Comparison of neuroprotective and neurorestorative capabilities of rasagiline and selegiline against lactacystin-induced nigrostriatal dopaminergic degeneration. J Neurochem 105:1970-1978 